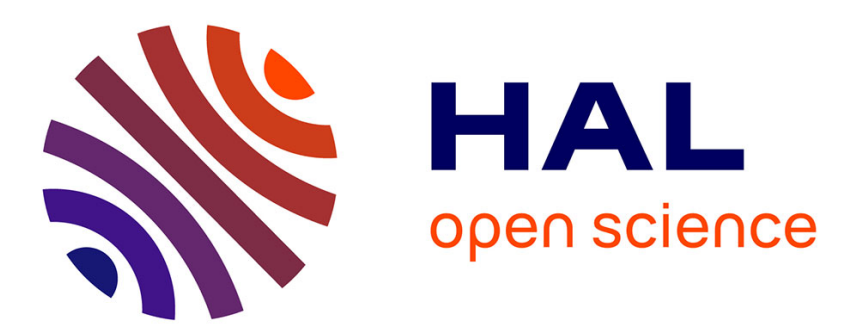

\title{
Results from FOPI on strangeness in nuclear matter at SIS energies
}

\author{
J-P. Alard, A. Andronic, R. Averbeck, V. Barret, Z. Basrak, N. Bastid, I. \\ Belyaev, A. Bendarag, G. Berek, R. Caplar, et al.
}

\section{- To cite this version:}

J-P. Alard, A. Andronic, R. Averbeck, V. Barret, Z. Basrak, et al.. Results from FOPI on strangeness in nuclear matter at SIS energies. Strangeness 2000 International Conference on Strangeness in Quark Matter 5, Jul 2000, Berkeley, United States. pp.267-273. in2p3-00007909

\section{HAL Id: in2p3-00007909 https://hal.in2p3.fr/in2p3-00007909}

Submitted on 19 Mar 2001

HAL is a multi-disciplinary open access archive for the deposit and dissemination of scientific research documents, whether they are published or not. The documents may come from teaching and research institutions in France or abroad, or from public or private research centers.
L'archive ouverte pluridisciplinaire HAL, est destinée au dépôt et à la diffusion de documents scientifiques de niveau recherche, publiés ou non, émanant des établissements d'enseignement et de recherche français ou étrangers, des laboratoires publics ou privés. 


\title{
Results from FOPI on strangeness in nuclear matter at SIS energies
}

\author{
P Crochet for the FOPI Collaboration: J P Alard ${ }^{1}$, A Andronic ${ }^{2,3}$, \\ R Averbeck ${ }^{3}$, V Barret ${ }^{1}, \mathbf{Z}$ Basrak $^{4}$, N Bastid ${ }^{1}$, I Belyaev ${ }^{5}$, A Bendarag ${ }^{1}$, \\ G Berek ${ }^{6}$, R Caplar ${ }^{4}$, N Cindro ${ }^{4}$, P Crochet $^{1}$, A Devismes ${ }^{3}$, P Dupieux ${ }^{1}$,

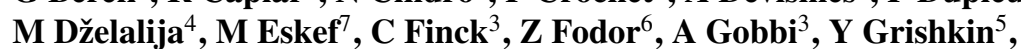 \\ O Hartmann ${ }^{3}$, N Herrmann ${ }^{3,7}$, K D Hildenbrand ${ }^{3}$, B Hong ${ }^{8}$, \\ J Kecskemeti $^{6}$, Y J Kim ${ }^{8}$, M Kirejczyk ${ }^{9}$, P Koczon ${ }^{3}$, M Korolija ${ }^{4}$,

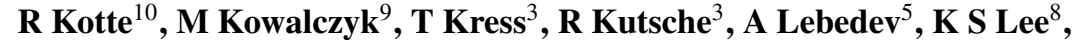 \\ Y Leifels $^{3,7}$, V Manko ${ }^{11}$, H Merlitz ${ }^{7}$, S Mohren ${ }^{7}$, D Moisa ${ }^{2}$, W Neubert ${ }^{10}$, \\ A Nianine ${ }^{11}$, D Pelte ${ }^{7}$, M Petrovici ${ }^{2}$, C Plettner ${ }^{10}$, F Rami $^{12}$, W Reisdorf ${ }^{3}$, \\ B de Schauenburg ${ }^{12}$, D Schüll ${ }^{3}$, Z Seres $^{6}$, B Sikora ${ }^{9}$, K S Sim $^{8}$, V Simion ${ }^{2}$, \\ K Siwek-Wilczyńska ${ }^{9}$, V Smolyankin ${ }^{5}$, A Somov ${ }^{5}$, M Stockmeier ${ }^{7}$, \\ G Stoicea $^{2}$, M Vasiliev ${ }^{11}$, P Wagner ${ }^{12}$, K Wiśniewski ${ }^{3,9}$, D Wohlfarth ${ }^{10}$, \\ J T Yang ${ }^{8}$, I Yushmanov ${ }^{11}$ and A Zhilin ${ }^{5}$

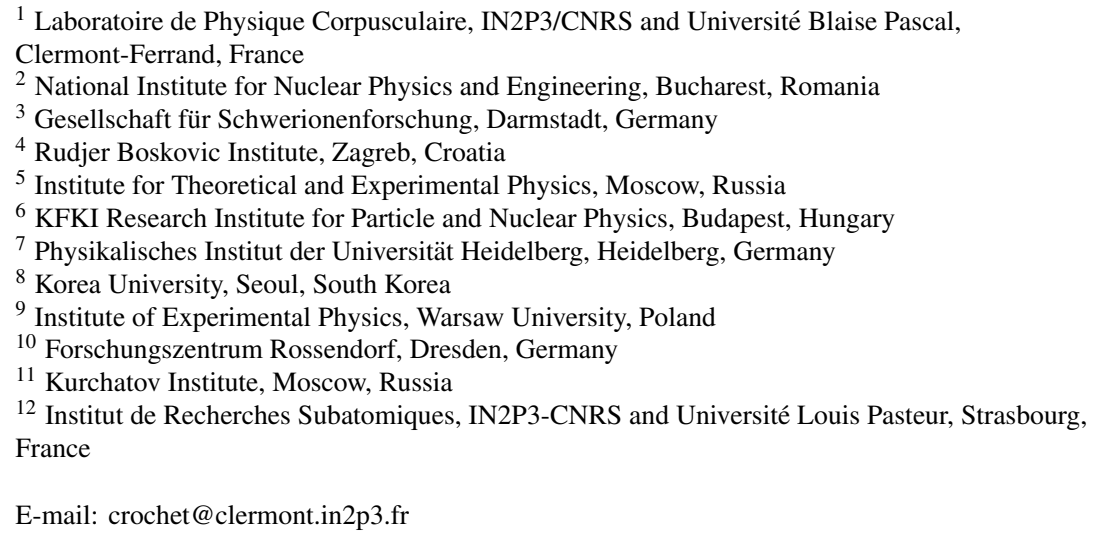

Received 2 November 2000

\begin{abstract}
Experimental data on charged kaon phase space distributions and strangeness sideward flow measured with the FOPI detector at SIS/GSI are presented. Comparisons are made with the predictions of transport models investigating the in-medium kaon-nucleon potential.

(Some figures in this article are in colour only in the electronic version; see www.iop.org)
\end{abstract}




\section{Introduction}

The possible modification of hadron masses and widths in hot and dense matter is a subject of considerable current interest. In particular, a large theoretical effort has been devoted to the investigations of the in-medium properties of kaons as they are important for understanding both chiral symmetry restoration and neutron star properties [1]. These studies, carried out using different approaches [2,3], converge qualitatively towards the common feature that in the nuclear medium the $K^{+}$mesons feel a weak repulsive potential, whereas the $K^{-}$mesons feel a strong attractive potential. Experimentally, the properties of kaons in a high-density medium can only be investigated by means of heavy-ion collisions. This is particularly relevant for beam energies of 1-2 AGeV since it corresponds to kaon production below threshold or closeto-threshold [1]. As the kaon in-medium potential results in a slightly increased $K^{+}$mass and a strongly reduced $K^{-}$mass, one expects to observe an enhanced $K^{-}$yield (its production being energetically much easier) and a reduced $K^{+}$yield (its production being energetically more difficult). On the other hand, the kaon potential should repel $K^{+}$from nucleons and attract $K^{-}$towards nucleons. This would influence the phase space populations by a widening (narrowing) of the $K^{+}\left(K^{-}\right)$transverse momentum and rapidity distributions. First signs of these effects have been observed very recently by the KaoS and the FOPI collaborations [4-6]. Substantial uncertainties about the strength of the in-medium potential, especially its momentum dependence [7,8], and about the in-medium cross sections [8] motivate further theoretical investigations as well as more detailed data. In this contribution we report on recent measurements on strangeness yield and sideward flow measured with the FOPI detector $[9,10]$ for the reactions $\mathrm{Ni}+\mathrm{Ni}$ at $1.93 \mathrm{AGeV}$ and $\mathrm{Ru}+\mathrm{Ru}$ at $1.69 \mathrm{AGeV}$.

\section{Pion and kaon yields}

The rapidity distribution of $K^{+}$in $\mathrm{Ni}+\mathrm{Ni}$ reactions at $1.93 \mathrm{AGeV}$ is shown in figure 1 . The data, from the FOPI and the KaoS collaborations, are compared with the predictions of various transport codes without (left) and with (right) in-medium kaon-nucleon potentials. The first situation corresponds to a standard calculation including binary collisions plus 'conventional' potentials (e.g. Coulomb potential). In the second scenario in-medium potentials are added. The discrepancies between the calculations in the left part of figure 1 demonstrate that the bare cross sections used in the models are not universal but are rather model-dependent. The inclusion of in-medium effects reduces the overall $K^{+}$yield leading to a good agreement with the data.

It is particularly important to mention that although all transport codes consistently reproduce the proton rapidity distributions in the SIS energy range $[13,14,16]$, the situation remains unclear concerning the pion yield. Indeed, as shown in figure 2, whereas the RBUU model reproduces the $\pi^{-}$yield consistently in small systems, most of the transport codes overestimate both the $\pi^{-}$and the $\pi^{+}$yield in large systems. Apparently the discrepancy is increasing with system size. This raises questions concerning the relevance of the previous conclusions on the kaon yield since, in the SIS beam energy range, kaon production originates mostly from multi-step processes for which pions play an important role.

\section{The $K^{-} / K^{+}$ratio}

The study of the $K^{-} / K^{+}$ratio is motivated by the following considerations. According to the theoretical predictions mentioned in the introduction, in-medium effects are more important for $K^{-}$than for $K^{+}$. However, since the $K^{-}$production yield at SIS energies is very small, 

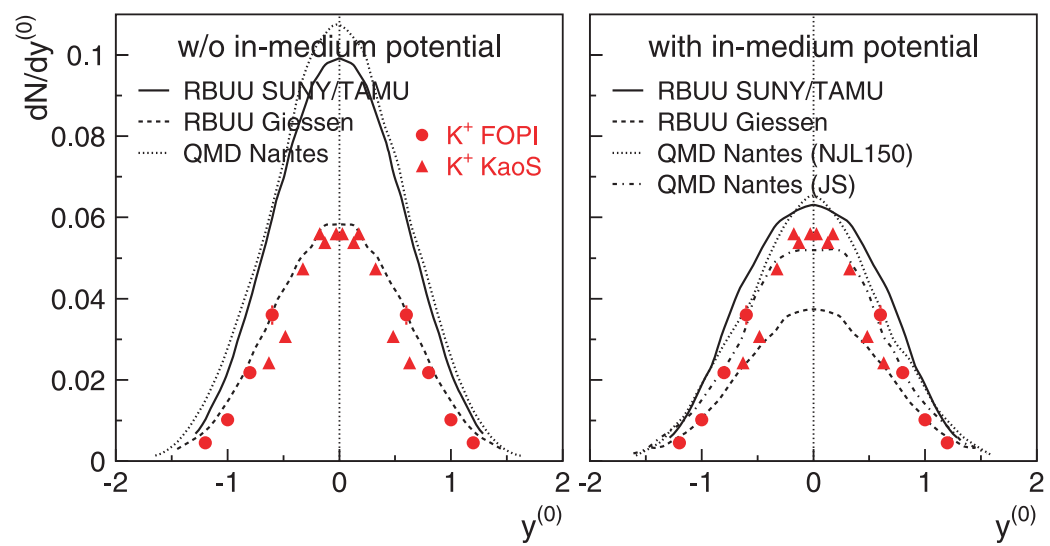

Figure 1. $K^{+}$rapidity distribution in central $\mathrm{Ni}+\mathrm{Ni}$ reactions at $1.93 \mathrm{AGeV}$. FOPI data (dots) and KaoS data (triangles) are compared with the predictions of various transport codes without (left) and with (right) the in-medium potential. FOPI and KaoS data are from [11,12], respectively. The calculations referred to SUNY/TAMU, Giessen and Nantes are from [13-15], respectively.
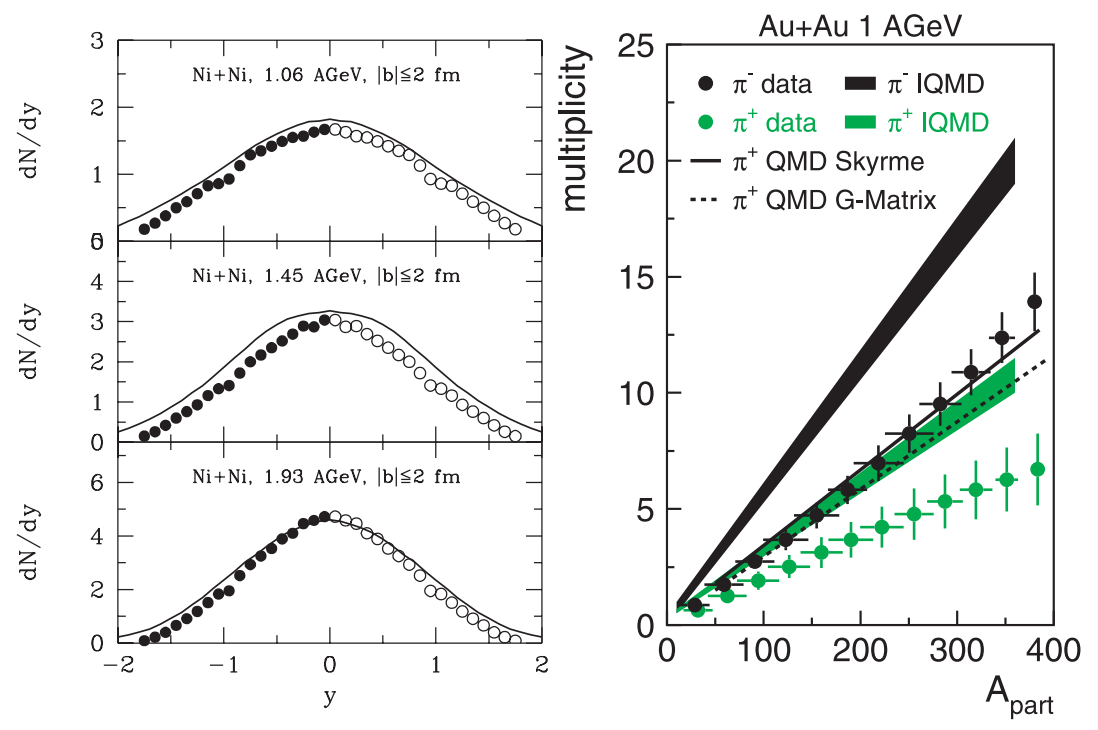

Figure 2. Left, rapidity distribution of $\pi^{-}$in central $\mathrm{Ni}+\mathrm{Ni}$ reactions at various beam energies. The data (dots) [16] are compared with the predictions of the RBUU model (taken from [13]). Right, charged pion multiplicity as a function of the number of participants in $\mathrm{Au}+\mathrm{Au}$ reactions at 1 AGeV. Data and IQMD calculations are from [17], QMD calculations are from [18].

the limited statistics do not allow one to examine the full $K^{-}$phase space distributions with a high accuracy. The study of the $K^{-} / K^{+}$ratio is meaningful and particularly interesting since by doing so statistical limitations and experimental detector inefficiencies cancel out to some extent. In addition, since in-medium effects act oppositely on $K^{-}$and $K^{+}$, their ratio should be most sensitive to these effects.

Figure 3 shows $K^{-} / K^{+}$ratio as a function of the centre-of-mass kinetic energy and rapidity. The transport code calculations shown in the figure predict, without in-medium effects, a rather flat behaviour of the ratio as a function of both $E_{\mathrm{cm}}^{\mathrm{kin}}$ and $y^{(0)}$. Oppositely, with in-medium 

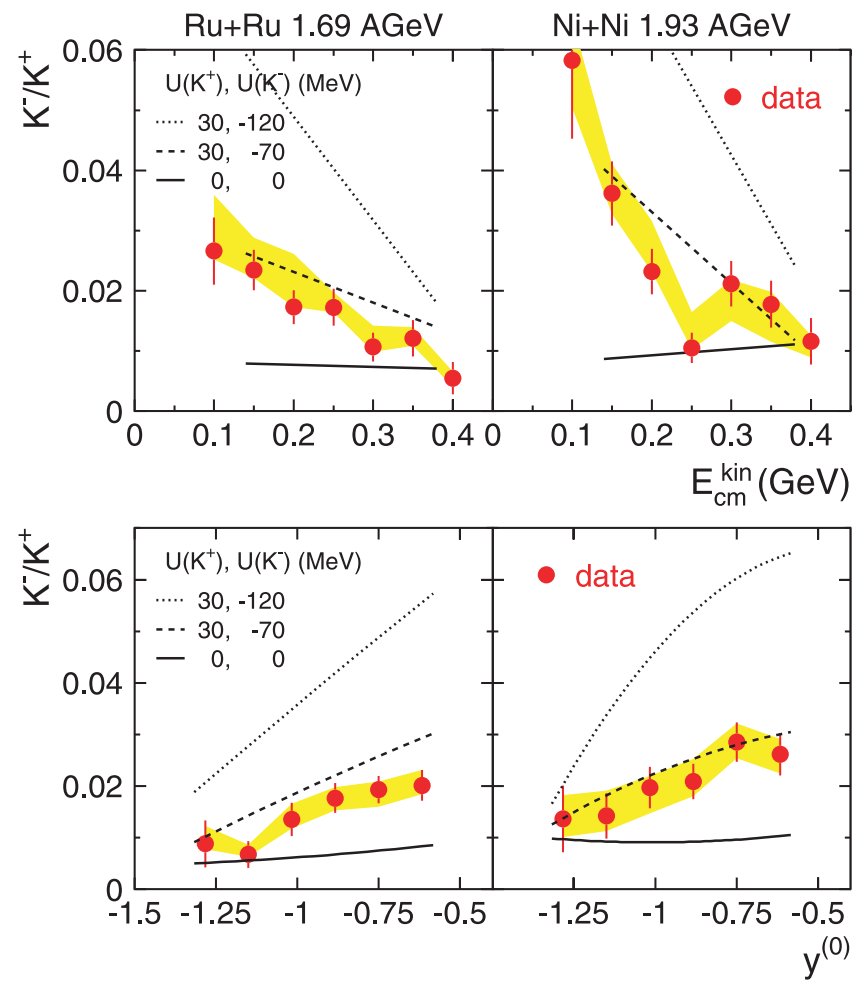

Figure 3. The $K^{-} / K^{+}$ratio as a function of the centre-of-mass kinetic energy (top) and rapidity (bottom) for central $\mathrm{Ru}+\mathrm{Ru}$ (left) and $\mathrm{Ni}+\mathrm{Ni}$ reactions (right). $y^{(0)}$ denotes the particle rapidity divided by the beam rapidity in the centre-of-mass system. In the upper figures, the data are extracted in the polar-angle range $150^{\circ}<\Theta_{\mathrm{cm}}<165^{\circ}$. The light grey shaded areas show the estimation of systematic uncertainties. The curves depict predictions of the RBUU model [14] with different strength of the in-medium (anti)kaon potentials at normal nuclear matter density (from [6]).

effects, a well defined maximum appears at low $E_{\mathrm{cm}}^{\mathrm{kin}}$ and towards mid-rapidity. It can be interpreted as the consequence of the interplay of three effects:

(a) with in-medium effects the yield of $K^{-}$is enhanced more than that of $K^{+}$is reduced;

(b) this is most pronounced at mid-rapidity where, thanks to the high baryon density, $K^{-}$are produced more abundantly; and

(c) the effective $K$-nucleon potential tends to push $K^{+}$away from nucleons (i.e. to large rapidities) and to attract $K^{-}$towards nucleons (i.e. to small rapidities).

The latter effect is also well seen in the kinetic energy dependence of the ratio since, due to the $K$-nucleon potential, $K^{+}$are pushed towards high momenta, whereas $K^{-}$are pulled towards low momenta. The data are nicely reproduced by the calculations only if in-medium potentials are taken into account.

\section{Strangeness sideward flow}

Collective flow effects of various ejectiles emerging from a heavy ion reaction have been studied extensively over the two last decades in both experimental data and transport-model 
theories $[19,20]$. In particular, the collective flow of kaons, both the in-plane component and the out-of-plane component [21], is recognized as a relevant observable to probe the kaon potential [22]. The first experimental data on strangeness sideward flow have been obtained by the FOPI collaboration in $\mathrm{Ni}+\mathrm{Ni}$ reactions at $1.93 \mathrm{AGeV}[23,24]$. Whereas the $\Lambda$ flow pattern is very similar to that of protons, the data show a vanishing $K^{+}$flow and $K_{\mathrm{S}}^{0}$ flow in the representation of the mean in-plane transverse momentum versus rapidity. The sensitivity of such data to in-medium effects is under intense debate. According to [25] the $K^{+}$data clearly support the existence of a repulsive $K^{+}$-nucleon mean field. According to [26, 27] the sensitivity of the observable to in-medium effects is found to be less pronounced but a slightly repulsive potential cannot be excluded from the comparison. On the other hand, the sensitivity of $K^{+}$sideward flow to in-medium effects was found in [7] to be washed out when a particular momentum dependence of the potential is included in the calculations. It was recently pointed out in [28] that the lifetime of nuclear resonances used in the models might be partially responsible for the magnitude of the $K^{+}$sideward flow as it strongly affects the kaon production characteristics.

In order to further elucidate these questions, we have investigated the transverse momentum dependence of the sideward flow in the $\mathrm{Ru}+\mathrm{Ru}$ and $\mathrm{Ni}+\mathrm{Ni}$ systems in a region of phase space which is free of any detector acceptance effects. Such a transverse momentum differential analysis does reveal more information than the transverse momentum integrated data where part of the effects are hidden. In addition, comparison of data to model predictions is straightforward since no corrections are necessary for the finite acceptance of the apparatus.

The $p_{t}$ dependence of $K^{+}, K_{\mathrm{S}}^{0}, \Lambda$ and proton sideward flow is shown in figure 4 for central $\mathrm{Ni}+\mathrm{Ni}$ reactions and central and semi-central $\mathrm{Ru}+\mathrm{Ru}$ reactions. It can be seen that the $K^{+}$flow pattern is totally different from that of protons. Protons have a negative $v_{1}$ for all $p_{t}$. Since the rapidity window used is located in the backward hemisphere, this means that protons are positively flowing whatever their $p_{t}$. In contrast, $K^{+}$have positive (negative) $v_{1}$ for low (high) $p_{t}$. In other words, $K^{+}$are negatively flowing (or antiflowing) at low $p_{t}$ and positively flowing at high $p_{t}$. We stress that, for the $\mathrm{Ni}+\mathrm{Ni}$ system, vanishing $K^{+}$flow was seen, if $p_{t}$-integrated data were used $[23,24]$. The same features are observed for the $\mathrm{Ru}+\mathrm{Ru}$ system. From the calculations shown in figure 4 , one can conclude that without in-medium potential the transport code fails to describe the low- $p_{t} K^{+}$antiflow phenomenon observed in the data. In contrast, when in-medium effects are taken into account the model reproduces quantitatively $K^{+}$experimental signals for both systems. The additional repulsive potential pushes $K^{+}$further away from the nucleons, therefore resulting in an anticorrelation between the $K^{+}$flow and the proton flow at low $p_{t}$

On the other hand, the model fails to consistently describe the proton sideward flow data in the considered target rapidity region, although reasonable agreement is found in the midrapidity region. This discrepancy is mostly due to an improper separation of free protons and bound nucleons in the spectator rapidity region [31], which seems to be a general problem of transport model calculations. A similar discrepancy has indeed been observed from the comparison of the same data and the predictions of the IQMD model [32] and is also exhibited by the RQMD model for $\mathrm{Au}+\mathrm{Au}$ reactions at $11 \mathrm{AGeV}$ [33]. This shows that a more definite interpretation of the $p_{t}$-differential flow data for nucleon needs further detailed investigations.

The $K_{\mathrm{S}}^{0}$ sideward flow pattern is found to be very similar to that of $K^{+}$. In particular, the same antiflow phenomenon is exhibited at low $p_{t}$. A similar antiflow pattern has also been observed for $K_{\mathrm{S}}^{0}$ in $\mathrm{Au}+\mathrm{Au}$ collisions at $6 \mathrm{AGeV}$ [34]. The $\Lambda$ sideward flow is systematically smaller than the proton flow. This trend, which is also observed by other experiments [34], supports, according to theoretical interpretations [35-37], a $\Lambda$ potential of two-thirds of the nucleon potential. 


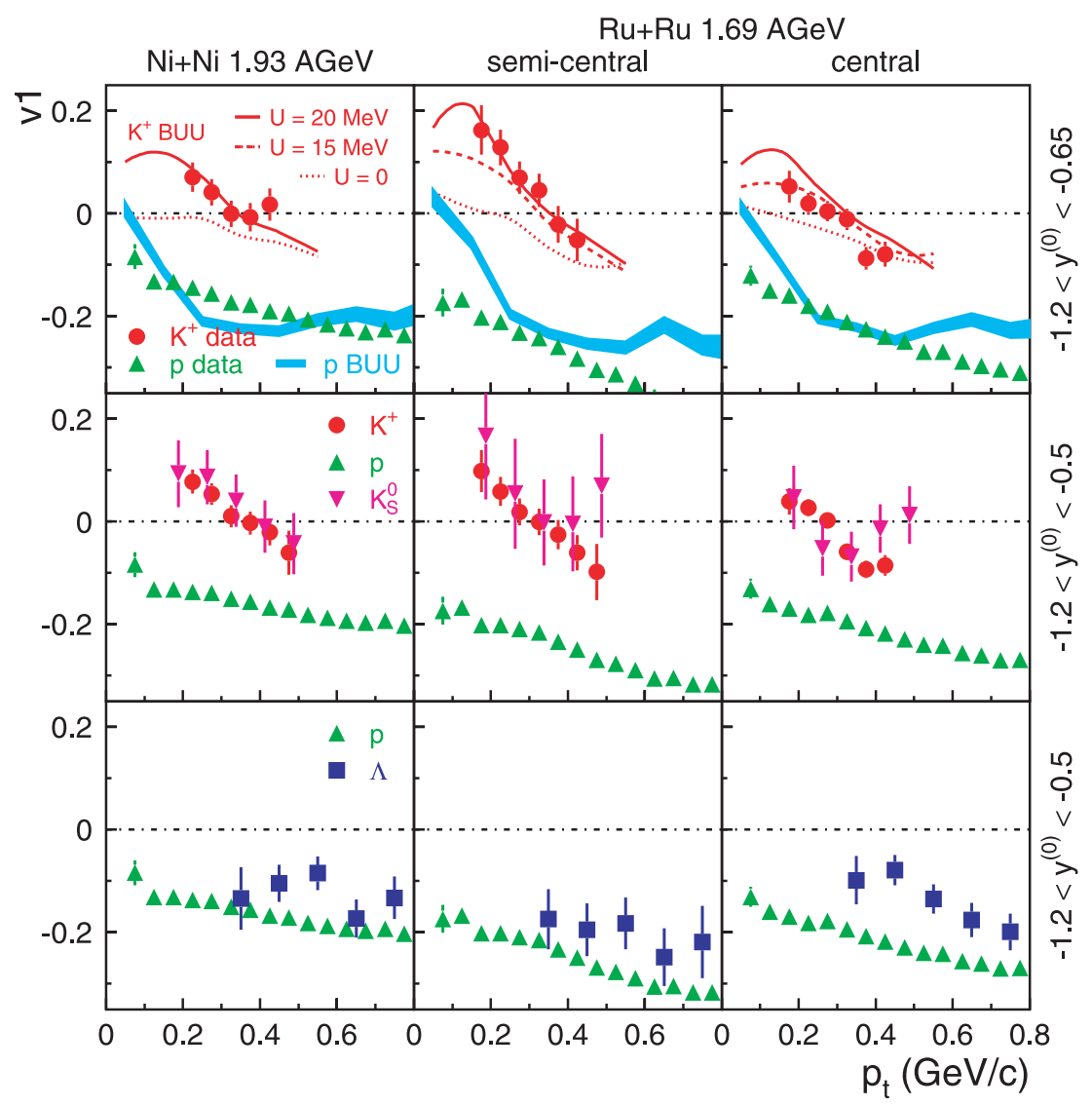

Figure 4. Transverse momentum dependence of $v_{1}$ for $K^{+}, K_{\mathrm{S}}^{0}, \Lambda$ and proton in central $\mathrm{Ni}+\mathrm{Ni}$ reactions (left) and in semi-central and central $\mathrm{Ru}+\mathrm{Ru}$ reactions (middle and right, respectively). The signal is extracted in the rapidity windows reported on the right of the figures. The $K^{+}$and proton data are compared with the predictions of the RBUU model [14]. Three different strength of the $K^{+}$potential at normal nuclear density are considered (from [29,30]).

\section{Conclusions}

Kaon phase space distributions and sideward flow have been measured in $\mathrm{Ni}+\mathrm{Ni}$ reactions at $1.93 \mathrm{AGeV}$ and $\mathrm{Ru}+\mathrm{Ru}$ reactions at $1.69 \mathrm{AGeV}$ with the FOPI detector at GSI. The data were compared with model predictions investigating in-medium modifications of kaon properties. The overall picture emerging from the comparison tends to favour the existence of a nonzero in-medium kaon-nucleon potential. However, before one can draw any final statement on the kaon data, one would have to carefully compare $\pi$ and baryon data with the same transport model calculations. Indeed, (a) in large systems, the charged $\pi$ multiplicity is systematically overestimated by transport models in the SIS beam energy range and (b) the proton $p_{t}$-differential flow data is not satisfactorily reproduced by these models in the spectator rapidity region. Further exciting possibilities will be offered soon with the upgrade of the FOPI detector [38]. This will allow one to study with better statistics and accuracy $K^{-}$s for which in-medium effects are expected to be much more pronounced. 


\section{References}

[1] For a review, see Ko C M et al 1997 Ann. Rev. Nucl. Part. Sci. 471

[2] Mao Get al 1999 Phys. Rev. C 593381

[3] Schaffner J et al 1997 Nucl. Phys. A 625325

[4] Barth R et al (KaoS collaboration) 1997 Phys. Rev. Lett. 784007

[5] Laue F et al (KaoS collaboration) 1999 Phys. Rev. Lett. 821640

[6] Wiśniewski K et al (FOPI collaboration) 2001 Eur. J. Phys. A at press

[7] Fuchs C et al 1998 Phys. Lett. B 434245

[8] Schaffner-Bielich J et al 2000 Nucl. Phys. A 669153

Schaffner-Bielich J 2001 J. Phys. G: Nucl. Part. Phys. 27

[9] Gobbi A et al (FOPI collaboration) 1993 Nucl. Instrum. Methods A 324156

[10] Ritmann J et al (FOPI collaboration) 1995 Nucl. Phys. B Proc. Suppl. 44708

[11] Best D et al (FOPI collaboration) 1997 Nucl. Phys. A 625307

[12] Menzel M et al (KaoS collaboration) 2000 GSI Annual Report 2000-01 47

[13] Li G Q et al 1997 Nucl. Phys. A 625372

[14] Cassing W and Bratkovskaya E L 1999 Phys. Rep. 30865

[15] Hartnack C and Aichelin J 2000 Proc. Int. Workshop 28th on Gross Properties of Nuclei and Nuclear Excitations, (Hirschegg) p 129

[16] Hong B et al (FOPI collaboration) 1998 Phys. Rev. C 57244

[17] Pelte D et al (FOPI collaboration) 1997 Z. Phys. A 357215

[18] Maheswari V S U et al 1998 Nucl. Phys. A 628669

[19] Reisdorf W and Ritter H G 1997 Ann. Rev. Nucl. Part. Sci. 47663

[20] Herrmann N et al 1999 Ann. Rev. Nucl. Part. Sci. 49581

[21] Shin Y (KaoS collaboration) 2001 J. Phys. G: Nucl. Part. Phys. 27275

[22] Ko C M 2001 J. Phys. G: Nucl. Part. Phys. 27327

[23] Ritman J et al (FOPI collaboration) 1995 Z. Phys. A 352355

[24] Ritman J et al (FOPI collaboration) 1997 GSI Annual Report 97-1 61

[25] Li G Q and Brown G E 1998 Nucl. Phys. A 636487

[26] Bratkovskaya E L et al 1997 Nucl. Phys. A 622593

[27] Wang Z S et al 1998 Nucl. Phys. A 628151

[28] David C et al 1999 Nucl. Phys. A 650358

[29] Crochet P et al (FOPI collaboration) 2000 Phys. Lett. B 4866

[30] Kutsche R et al (FOPI collaboration) 2000 GSI Annual Report 2000-1 42

[31] Cassing W and Bratkovskaya E L Private communication

[32] Crochet P et al (FOPI collaboration) 1999 GSI Annual Report 99-1 49

[33] Barrette J et al (E887 collaboration) 1997 Phys. Rev. C 563454

[34] Chung P et al (E895 collaboration) 1999 J. Phys. G: Nucl. Part. Phys. 25255

[35] Li G Q and Ko C M 1996 Phys. Rev. C 541897

[36] Li G Q and Brown G E 1998 Nucl. Phys. A 636487

[37] Wang Z S et al 1999 Nucl. Phys. A 645177

[38] FOPI collaboration 2000 GSI Annual Report 2000-1 215 